\title{
pneumatic Muscle Actuators for Humanoid applications - Sensor and Valve Integration
}

\author{
S. Davis and Darwin G. Caldwell \\ Centre for Robotics and Automation \\ University of Salford \\ Manchester, M5 4WT, UK \\ s.davis@salford.ac.uk \\ d.g.caldwell@salford.ac.uk
}

\begin{abstract}
The development of humanoid robots places demands on designers unlike those of traditional robots. The close operation of such systems with humans means safety is a primary concern. As a result biomimetic actuators that duplicate the safe interaction between organic life forms are becoming increasingly popular. The pneumatic Muscle Actuator is one such system having performance characteristics equal to or better than that of organic muscle. One drawback of the actuator is the quantity of external hardware needed to drive it, particularly valves and sensors. This paper examines how this hardware affects actuator power/weight, force/weight, volume/weight and electrical power consumption. It is shown that by redesigning the valve system these factors can be improved and by integrating sensors into the pMA a 'plug and play' actuator which can easily be mounted to robotic structures can be produced.
\end{abstract}

\section{INTRODUCTION}

As interest in developing humanoid robots has increased there has been a reassessment of the technologies and principles used in robot design. Traditionally robots have been highly massive and stiff, making them unsuited to close human interaction. Due to the potential operating environments of humanoid robots, human safety must be a primary design concern. One approach to this problem, which is seeing increasing appeal, is to replicate the safe interaction between organic life forms. This approach is typically referred to as biomimetics or biologically inspired design.

This principle has led designers to re-examine their choice of materials but particularly actuators, with increasing interest in systems with performance characteristics similar to organic muscle. One such system is the braided pneumatic Muscle Actuators (pMAs) derived from the McKibben muscle, pioneered in the 1960's [1]. When studying the performance of pMA it can be seen that it equals, or betters, biological muscle in a number of categories. Both systems have comparable displacement, efficiency, bandwidth [2],[3] and control characteristics [4] as well as both having compliant operation [5]. The pneumatic system, however, offers vastly superior power/weight and force/cross sectional area performance than the organic system [5] making it ideally suited to humanoid applications [6]. A potential drawback of the pMA, however, is the quantity of peripheral non-integrated hardware. This includes both external valves and sensors and in this structure the biomimetic model that can so successfully be ascribed to the pMA breaks down. These external structures also add additional weight, volume, and possible power consumption and should be minimised or eliminated.

This paper further develops the soft biomimetic concepts of pMA enhancing performance by minimising the effect of the added hardware on power/weight, force/weight, volume/weight and electrical power consumption. Within this paper a new valving systems is described that is completely integrated into the structure of the current muscles. Similar work will show a multi-functional sensing (force, pressure and displacement) design that is also embedded within the muscle allowing the production of an integrated actuator. This 'plug and play' actuator can be mounted to robotic structures without the need for additional hardware providing a potential solution to the human-robot interaction paradigms that are increasingly being developed for humanoid applications.

\section{VALVES}

When discussing power/weight, force/weight and volume/weight ratios and the energy efficiency of an actuator it is usual to consider only the actuator itself and no mention is made of external components, such as drive circuitry, heatsinks or in the case of pMAs the valves used to supply the actuators with air. These valves irrespective of type will have a mass and a power consumption which for completeness should be included in any performance and efficiency claims. It is equally important that these features are identified for all other actuators. Pneumatic systems do indeed need a compressor to generate compressed air but equally electric systems need an electrical generator or batteries. Indeed one of the major reasons electric motors have seen only limited success in mobile applications is due to the high mass of electrical energy storage devices and the time to recharge. Pneumatic technology in contrast tends to suffer less from this problem as gas can be stored and created much more efficiently and can be fully recharged in seconds/minutes. At the same time much of the potential benefit of pMA technology can be completely lost if the electrical power consumption of the valves is high. 
The most common form of valve used in pneumatics is the solenoid valve. This is primarily due to its low cost, but a solenoid is heavy (usually having an iron core) and uses a large amount of electrical current and therefore is not ideally suited to use with pneumatic Muscle Actuators as this negates the actuator's power/weight benefits.

The valves used in previous projects [5][8][11] and in the pneumatic biped described in [6] are the MATRIX 750 [7] series and provide reasonable flow, power and weight characteristics. However they are far from an ideal solution. The valves are available in blocks of eight identical ports each with a single solenoid valve. The mass of the valve block is $0.34 \mathrm{~kg}$ [7], which is equivalent to $86 \mathrm{~g}$ per muscle as two ports are needed to control a single muscle. The MATRIX valve has a flow rate of $100 \mathrm{l} / \mathrm{min}$ at $600 \mathrm{kPa}$ and electrical power consumption per channel of $1.9 \mathrm{~W}$ [7]. However it can easily be seen that when using multiple muscle systems both valve mass and power consumption soon becomes a major obstacle to design and for this reason the valves are used in the pneumatic biped were remotely located with air being supplied to the robot via an umbilical.

\section{A. Valve Weight}

The power to weight ratio of a typical pneumatic Muscle Actuator is $500-2 \mathrm{~kW} / \mathrm{Kg}$ [5]. However, if the valve weight of $86 \mathrm{~g}$ determined above is included in this the power/weight ratio is reduced by approximately one third for a typical actuator $(50 \mathrm{~cm}$ long with a diameter of $50 \mathrm{~mm})$ weighing $150 \mathrm{~g}$.

The end-caps of the muscles are usually formed from a solid material (aluminium, nylon, or brass) onto which the rubber bladder and braided shell are clamped using steel clips. These caps are drilled and tapped and have fittings to allow air to enter the muscle. As the end-caps form an integral part of the pMA their mass is always included in any calculations. It is therefore essential that the mass of the valve be as low as possible. A simple way of reducing/eliminating this valve mass is to incorporate it into the end-cap.

In addition to reducing the overall mass of the system by locating the valve in the muscle end-cap the pressure losses present in the tube between valve and muscle are also eliminated. As the Matrix valves were not available in compact single valves packages an alternative valve solution was sought.

A commercially available solenoid valve ( $\mathrm{KV}$ Automation Systems, Microsol) was selected for this task. This valve is $47 \mathrm{~mm} \times 15 \mathrm{~mm} \times 21 \mathrm{~mm}$ and weighs $30 \mathrm{~g}$. The valve was mounted in the end-cap by first attaching pipes to the solenoid valve to transfer the inlet and outlet port from the side of the valve to the top and bottom. This was then placed between a two piece end-cap shell as can be seen in Figure 1 and then the shell was filled with an epoxy resin to form a solid end-cap with the valve located at it's centre. Two identical end-cap mounted valves are required to enable both filling and venting of the muscle to be controlled.

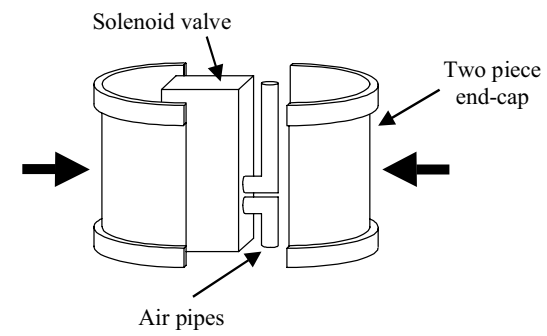

Figure 1 -Solenoid valve mounted in the centre of a two piece end-cap.

The overall mass of the new valve integrated muscle was $174 \mathrm{~g}$ with a peak air flow at $400 \mathrm{kPa}$ of approximately $50 \mathrm{l} / \mathrm{min}$ and a power consumption of $1.2 \mathrm{~W}$ per valve. This can be compared to the mass of a typical muscle and valve (MATRIX 750 ) of $236 \mathrm{~g}$. The new muscle with integrated valves offers a $26 \%$ weight saving. Despite this improvement the $30 \%$ reduction in flow (Matrix 750 has flow of $701 / \mathrm{min}$ at $400 \mathrm{kPa}$ ) caused by using a valve small enough to be mounted in the end-cap means the overall power to weight ratio remains almost unchanged. It might therefore seem that this renders the modification worthless, however, when considering the actuators force/weight ratio this can be seen to increase by $26 \%$. The result therefore shows that if actuator speed is less of a concern, the reduction in mass caused by using the valve to form part of the actuator end-cap is indeed beneficial.

\section{B. Valve Power Requirement}

Although the mass of the valve was a significant concern a more pressing problem was the electrical power consumption and the need for mains supplies or battery storage. Costa et al [8] demonstrated how the power consumption of the MATRIX valves (driven by a specially developed board) could be dramatically reduced. Despite this, however, when many valves are used the total power requirement rapidly becomes prohibitive.

To reduce the power consumption of the control hardware still further alternative forms of valve were considered. Piezoelectric valves were found to provide an excellent solution due to their incredibly low power requirements. Such valves use piezoelectric bimorphs, to control the flow of air though a valve orifice. One such valve is the Joucomatic Piezotronic [9] valve which has a power consumption of just $24 \mathrm{~mW}$. However, due to the comparatively low force generated by a piezoelectric bimorph the valve orifices must be small and this results in low air flow rates. This is evident in the Piezotronic valve which has a flow rate of just $71 / \mathrm{min}$ which is obviously of no use except in the smallest of actuators.

One way to overcome this problem is to use a piezoelectric valve to control the flow of air to a pneumatically operated switch known as a poppet. This switch would in turn control the flow of air from a much higher flow channel to the actuator. This type of system can be considered as a pneumatic 
transistor and is completely analogous to an electrical system. The base current $i_{B}$ is analogous to the flow from the piezoelectric valve and the collector current $\mathrm{i}_{\mathrm{C}}$ is similar to the main flow channel through the poppet valve.

The Hoerbiger P8 poppet valve [10] is a commercially available valve of this type. The valve has a flow rate of 110 $1 / \mathrm{min}$ at $600 \mathrm{kPa}$ which is comparable with the Matrix valves currently used. However, the power requirement of the valve is considerably lower at just $5.8 \mathrm{~mW}$. However the main drawback of the valve is its high mass. Each Hoerbiger valve has a mass of $120 \mathrm{~g}$ which means that each muscle would require $240 \mathrm{~g}$ of associated valves which is considerable when compared to the $86 \mathrm{~g}$ of Matrix valves.

\section{Integrated Poppet Valve}

Having identified potential valve solutions it was clear that the goal should be to combine the power saving performance offered by the piezoelectric based valves with the weight saving achieved by mounting the valves in the endcaps. To achieve this a new actuator endcap was designed which incorporates a poppet valve as can be seen in Figure 2. The poppet valve produced has a diameter of $30 \mathrm{~mm}$, a length of $38 \mathrm{~mm}$, an orifice diameter of $2 \mathrm{~mm}$ and a weight of $66 \mathrm{~g}$. The valve chosen to drive the poppet was the Joucomatic Piezotronic valve, detailed earlier, due to its low mass $(25 \mathrm{~g})$ and low power requirement $(24 \mathrm{~mW})$. The total weight of the test muscle created including the two Piezotronic valves and the two poppets required to control both filling and venting was just $255 \mathrm{~g}$.

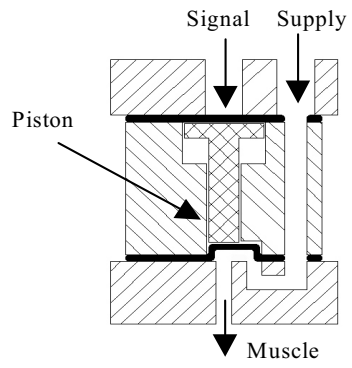

(a) - Valve Open

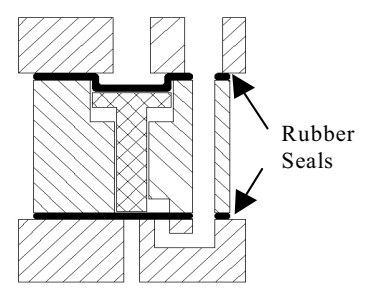

(b) - Valve Closed
Figure 2 - Muscle end-cap with integrated poppet valve shown in both open and close configuration.

When pressure is applied to the supply input, this impinges on the bottom end of the piston causing it to rise and allowing the air supply to enter the muscle as can be seen in Figure 2(a). The output from the piezoelectric valve is applied to the signal input as shown in Figure 2. If this pressure is equal to the supply pressure the piston moves to the valve closed position. This is because the force generated on the upper face of the piston is greater than that on the bottom due to its larger area. When in its lower position the piston blocks the flow of air from the supply to the muscle as can be seen in Figure 2(b). In this configuration the poppet valve only allows air to be applied to the muscle. To permit venting a second valve is located in the opposite end-cap. The poppet design in this second valve is identical to the one shown above, however, instead of applying a pressure at the supply port this is left open to the atmosphere. In its non-activated state, air flows from the muscle to atmosphere, however, when a signal pressure is applied this path is closed and venting is prevented.

To allow the performance of the valve to be tested a rig was produced which consisting of a single actuator similar in dimension to those used in the calf of the pneumatic biped described in [6]. The rig allowed both muscle pressure and displacement to be controlled within a closed loop using the new valve design. Figure 3 shows the isometric performance of the muscle at one set length $(0.35 \mathrm{~m})$ for a range of pressure step inputs. The same test was repeated on an identical sized actuator using the Matrix valves in order to provide a performance comparison.

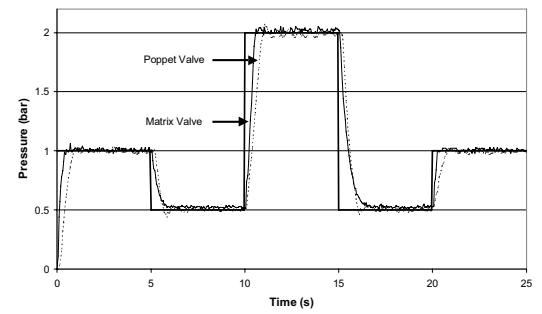

Figure 3 - Step pressure response for Matrix and poppet valves at a range of arbitrarily selected pressures.

It can be seen that the pressure profile tracks the input signal demand for both valve types and can be controlled accurately although the response time of the poppet valve is slightly slower (longer delays) than that of the matrix valve. There are two factors that contribute to this difference.

Firstly the switching time of the poppet valve is longer than that of the Matrix valve. The time taken for a step change in input to appear as a change in actuator pressure was measured to be $\approx 80 \mathrm{~ms}$ for the poppet valve compared with $\approx 50 \mathrm{~ms}$ for the Matrix valve.

The second factor which effects the response of the actuator is the airflow rate through the valve. It is difficult to measure this due to the probability of the flow being turbulent but by studying the experimental data for the two valves it is possible to determine how the poppet valve performs in comparison with the known performance data for the Matrix valve.

When the volume of the actuator is constant, as is the case in isometric testing, the volume of air present inside the muscle is proportional to its pressure. It therefore follows that the gradient of a plot showing pressure variation over time, for a constant volume actuator, will be proportional to the air flow rate. So by simply comparing the gradients of the two data lines it is possible to determine the comparative performance. Using this method it was determined that the flow rate through the new poppet valve was approximately two thirds the flow of the Matrix valve.

In practical applications the actuators are not only required to 
respond to step inputs but should also be able to track inputs. Figure 4 shows the displacement response of the actuator with the poppet valve when attempting to track a sinusoidal input.
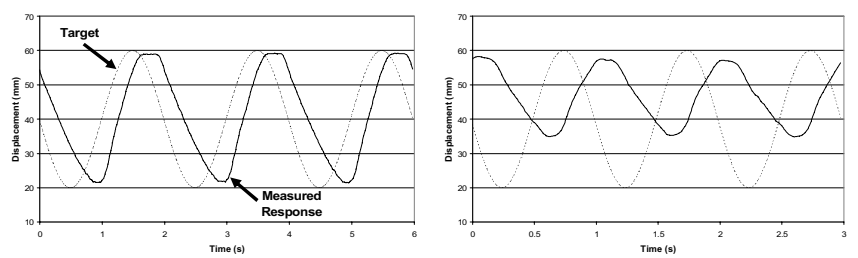

Figure 4 - Poppet valve tracing 0.5 and $1 \mathrm{~Hz}$ sinusoidal input (dashed line is target displacement).

It can be seen that the output displacement of the valve tracks the input reasonably well although the output is not a true sinusoid. At both frequencies the output appears sinusoidal as the muscle contracts but during relaxation the profile tracking is less accurate. The reason for this may be due to the pressure that returns the poppet to its 'valve open' position. In the filling valve this pressure is a combination of the supply pressure and the internal muscle pressure as seen in Figure 2. However, the venting valve has no supply pressure and so the returning force is only generated by the pressure inside the muscle. As the pressure in the muscle reduces during venting this force will also reduce and the dynamic performance of the poppet will change.

It can be seen that as the frequency of the input is increased the amplitude of the output reduces (bandwidth limitations) as would be expected. However, the midpoint of the output does not match the midpoint of the input instead it tends towards the maximum displacement position. The reason for this is due to the fact that the air flow rate through the fill valve is higher than that through the venting valve meaning the muscle can be pressurised faster than it can be vented. This is because during filling, air is forced into the muscle by the supply pressure $(500 \mathrm{kPa})$ but during venting the pressure which expels the air is only the reducing internal muscle pressure, which is considerably lower.

Also it can be seen that some of the output peaks have flat tops. This indicates that there is a delay between the venting valve receiving its signal to open and it actually opening. This may be caused by stiction between the rubber valve and the aluminium valve body.

When compared to the Matrix valve the new poppet valve has comparable mass but the flow rate through the valve is approximately $30 \%$ less. However, the advantages of this valve are that it uses only $1.2 \%$ of the electrical power of the Matrix Valve and is much more compact, as an external valve block is no longer required.

\section{SENSING}

When controlling the actuators various forms of sensor data can be required, the most common being muscle pressure, muscle force and muscle contraction.

\section{A. Pressure Sensing}

There are two main concerns associated with pressure sensors. Firstly, sensors are often located remotely from the actuators with an air line transmitting pressure from the actuator to the sensors [11]. This results in a discrepancy between the pressure read by the sensors and the actual pressure in the actuator. The size of this discrepancy is dependant on the nature of the connecting pipe as shown by Poiseuille's Law:

$\frac{\Delta P}{Q}=\frac{8 \cdot L \cdot \eta}{\pi \cdot r^{4}}$

Where $\Delta P$ is the pressure loss along a pipe of length $\mathrm{L}$ and inner radius $r$ at a fluid flow rate of $Q$ and where $\eta$ is the viscosity of the fluid.

It is apparent that the loss of pressure is dependant upon the length of the pipe used to transmit air to the sensor but much more crucially on the radius of the tube. The robot described by Davis et al [11] used remotely located pressure sensors connected to the actuators by pipes of typical length $0.5 \mathrm{~m}$ and inner diameter $2 \mathrm{~mm}$. The valves used to power the actuators have a maximum flow rate of $1.5 \mathrm{l} / \mathrm{s}$ and the viscosity of air at $25^{\circ} \mathrm{C}$ is $1.9 \times 10^{5} \mathrm{Ns} / \mathrm{m}^{2}$. It can therefore be calculated that the pressure measured by the sensor is approximately $35 \mathrm{kPa}$ less than the actual pressure in the actuator. The dynamic nature of the pressure readings means that any compensation for the error is problematic.

If larger diameter pipes are used this problem is reduced. For example the peak pressure loss in the tube is reduced to just $2 \mathrm{kPa}$ by increasing pipe diameters from 2 to $4 \mathrm{~mm}$. However, this solution can create problems with space particularly with the increased stiffness of the piping.

When low numbers of actuators are used on a system the additional pipes needed for the pressure sensors do not present a serious problem, however, with systems consisting of many 10 s of actuators the volume occupied soon adds up and increases the size, and to a lesser extent, weight of the overall system.

A solution to both of these problems is to locate the pressure sensors inside the actuators and this was demonstrated by including a piezoelectric pressure sensor in the actuator endcap as seen in Figure 5. The pressure losses caused by the pipes are eliminated and only a single pipe is required between the control valve and the actuator. An electrical connection now needs to be made with the muscle to allow sensor signals to be fed to the control hardware. However, these cables are smaller and more flexible than the air pipes used with the remote sensors and therefore require less space which could be further reduced by multiplexing or the use of a bus structure. 


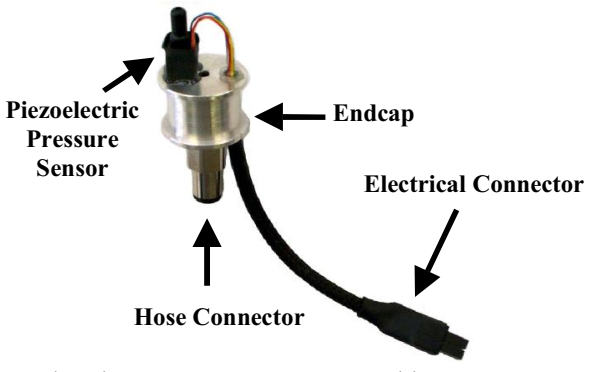

Figure 5 - Piezoelectric pressure sensor mounted in an actuator endcap.

\section{B. Force Sensing}

In some robotic systems force sensing is desirable. Force sensing has been combined with braided pneumatic Muscle Actuators in systems such as the exoskeleton described by Tsagarakis et al [12] where joint torques are measured by strain gauges mounted within pulleys. Although successful this technique removes/reduces one of the major advantages of the pMA technology which is the use of rapid low tech construction as precise machining is needed to produce pulleys with spokes onto which sensors can be mounted.

This problem can be overcome by the inclusion of force sensors on the actuators themselves. Each muscle endcap is fitted with a cantilever onto which strain gauges are bonded, as can be seen in Figure 6.

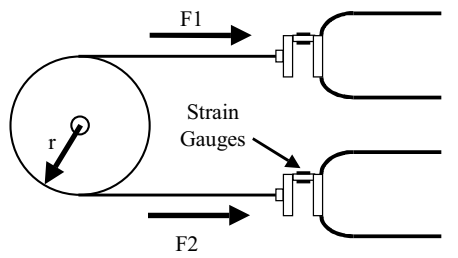

Figure 6 - Two muscles with force sensors allowing joint torque to be calculated.

The use of prefabricated actuators with force sensors again allows the possibility for rapid construction as sensors do not need to be included in the mechanical design. Force/torque control [11] of joints is still possible as actuator force data can be combined with the known physical structure of any pulley to determine a torque.

\section{Displacement Sensing}

Similar to the force sensors, the inclusion of joint position sensors makes system design more complex. This can again be remedied by including sensors that measure the length of the actuator within the basic actuator construction. From this data angular joint positions can be calculated simply in software.

Traditional linear sensors such as Linear Variable Differential Transformers (LVDT) tend to be rigid and highly expensive which detract from the actuators suitability to low cost construction, therefore a number of alternative low cost solutions were tested.

\section{Calculation}

From the actuator models it is clear that if two of the variables pressure, force and length are known then it is possible to calculate the third. Therefore, from the measured force and pressure data it was possible to calculate the length of the actuator, however, even with the most accurate model described in [2] errors of up to $5 \%$ of displacement were obtained during dynamic testing. This was unacceptably high as it would limit the precision of the system.

\section{Strain Gauge}

Hooke's law states that the force generated by a spring is proportional to the product of a spring constant $\mathrm{K}$ and the extension of the spring. It therefore follows that for a spring of known spring constant it is possible to determine the extension by measuring the force it generates. This system was tested as a method of measuring actuator displacement as can be seen in Figure 7. A spring was placed between the two endcaps of the actuator with one end being attached to a strain gauge based load cell capable of accurately measuring the force generated by the spring. As the actuator contracts the spring becomes shorter and therefore the force it produces decreases.

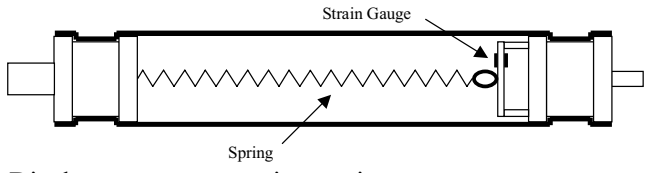

Figure 7 - Displacement sensor using strain gauge.

Figure 8 shows the results obtained from the sensors at a range of static displacements. The sensor was found to only be accurate to $\pm 2 \mathrm{~mm}$ which was not of high enough resolution for practical applications.

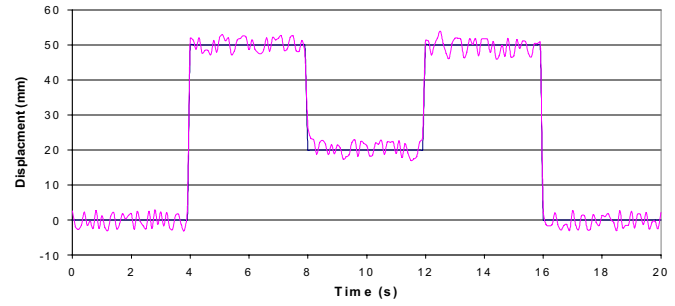

Figure 8 - Practical measurements observed from strain gauge sensor at three arbitrarily selected displacements.

Further problems exist with this method which means it is not practical. The weight of the spring generates an additional force on the load cell and as the orientation of the actuator changes the degree that this affects the displacement reading varies.

\section{Rotary potentiometer}

The next solution tested uses a cable, tensioned by a spring, that passes from the grounded end of the actuator over a pulley at the remote end and back to the grounded end as can be seen in Figure 9. As the actuator contracts the cable causes the pulley to rotate and the potentiometer attached to it records the change in displacement. The maximum number of turns in a typical multi-turn potentiometers is ten, meaning the maximum displacement the sensor can measure is ten times the circumference of the pulley. The diameter of the pulley is also limited, with its maximum diameter being the minimum internal diameter of the actuator. This means this sensor is not 
well suited to operation inside the actuator. An incremental encoder would provide unlimited displacement measurement, however the lack of absolute position data makes it unsuited to the required task.

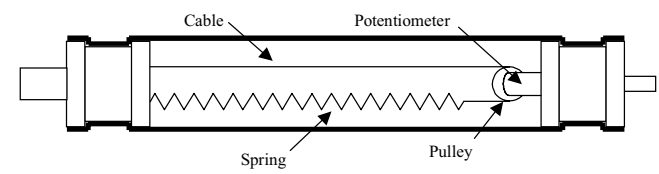

Figure 9 - Displacement sensor using rotary potentiometer.

\section{Resistance Wire}

The final solution tested used a length of resistance wire placed between the two endcaps of the actuator and again tensioned by a spring as can be seen in Figure 10. A set of brushes make contact with the resistance wire and allow a voltage to be measured between one end of the wire and the point where the brush is in contact. The brushes are mounted on a cable which is also tensioned by a spring and this therefore keeps the brushes a known distance from the end of the actuator, without the need for a rigid support structure. As the actuator contracts so the length of resistance wire conducting current (V) changes and from this an absolute displacement value can be determined.

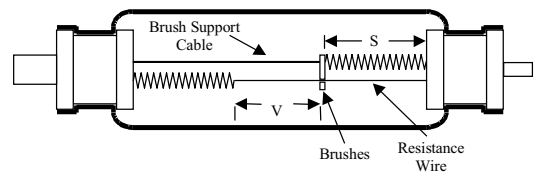

Figure 10 - Displacement sensor using resistance wire and brushes. Springs are used to keep the sensor in position.

The sensor was tested inside the same actuator as in the previous section and the results can be seen in Figure 11. The sensor proved accurate to $<0.5 \mathrm{~mm}$ which is considerably more accurate that the strain gauge based sensor system.

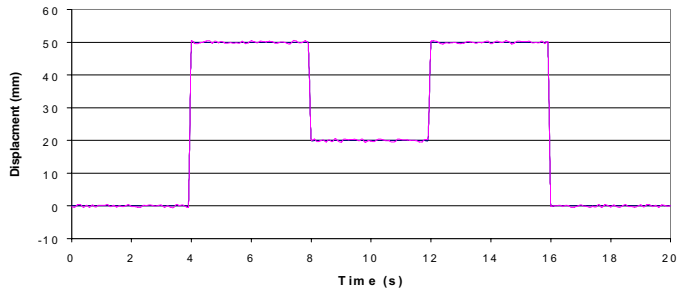

Figure 11 - Repeated displacement results using the resistance wire sensor.

\section{Sensor Integrated Muscle (Smart Muscle)}

As described previously the nature of the actuators allows for rapid system construction however when external sensors are required this ability is reduced. To overcome this it may be desirable to produce a 'plug and play' actuator which contains each of the required sensors. This would enable designers to rapidly produce robotic structures knowing that they did not need to design and fit sensors.

Each sensor is located inside the actuator and output signals are fed to control hardware via a common electrical connection located at the remote end of the actuator.
It was shown that a compact actuator/sensory assembly could be produced without any significant effect on the operation of the actuator.

\section{CONCLUSIONS}

This paper has considered the peripheral hardware required when pneumatic Muscle Actuators are being controlled. This consists of valves to control the flow of air to the actuators and sensors used to measure their performance.

The valves used to control the flow of air is rarely considered in power/weight, force/weight, volume/weight and power consumption calculations, however, clearly for completeness this should be included. Obviously to achieve peak performance the weight and power requirements of the valves used should be as low as possible. It has been suggested that in order to keep this mass low the valve should be mounted in the end-cap of the muscle thereby using the body of the valve to provide the shape and function of the end-cap. A number of solutions have been presented on this theme which have been shown to improve actuator force/weight by $26 \%$, reduce the electrical power requirement by $98.8 \%$ and reduce the overall volume of the peripheral hardware. At this time no tests have been conduced regarding the operational life of the valves and this will form vital future work.

Typically during control applications actuator displacement, pressure and force data are required. It has been shown that each of these can be measured by sensors incorporated into the actuator. This has a number of advantages including improved sensing accuracy and the possibility of developing a integrated 'plug and play' muscle consisting of both valves and sensors which can easily be mounted to a robotic platform. This removes the need for additional external sensing and therefore reduces system construction times.

\section{REFERENCES}

[1] H.F. Schulte. "The Characteristics of the McKibben Artificial Muscle". In the Application of External Power in Prosthetics and Orthetics. 1962.

[2] S. Davis, N. Tsagarakis, J. Canderle and D.G. Caldwell. "Enhanced Modelling and Performance in Braided pneumatic Muscle Actuators". International Journal of Robotics Research, Volume 22, Issue 3. 2003.

[3] D.A. Kingsley, R.D. Quinn. Fatigue Life and Frequency Response of Braided Pneumatic Actuators. IEEE ICRA. Washington, USA, 2002.

[4] D.G.Caldwell, G.A.Medrano-Cerda, and M.J. Goodwin, "Control of Pneumatic Muscle Actuators", IEEE Control Systems Journal, Feb. 95.

[5] D.G. Caldwell, N. Tsagarakis, G.A. Medrano-Cerda. "Bio-mimetic Actuators: Polymeric Pseudo Muscular Actuators for Biological Emulation". Mechatronics. 2000.

[6] P. Artrit, D.G. Caldwell, "Single Support Balancing of a Pneumatic Biped" CLAWAR 2001. Karlsruhe, Germany, 2001.

[7] "Matrix Catalogue". Matrix Italy.

[8] N. Costa, P. Artrit, And D. G. Caldwell. "Soft Interfaces For A Humanoid Robot - Karate Robot”. CLAWAR 2002. France. 2002.

[9] Asco Joucomatic Component Catalogue. 2002.

[10] Hoerbiger Orgia. Pneumatic Piezo Catalogue. October 2001.

[11] S. Davis, P. Tresadern, J. Canderle, N.G. Tsagarakis, P. Dodd, and Darwin G. Caldwell. "The Biomimetic Design of 'Soft' Mechatronic Systems". IEEE ICAR. Coimbra, Portugal. June/July 2003.

[12] N. Tsagarakis, D.G. Caldwell, G.A. Medrano-Cerda. "A 7 dof pneumatic Muscle Actuator (pMA) powered Exoskeleton". 8th IEEE Int Workshop on Robot and Human Interaction. Pisa, Italy. 1999. 